\title{
Risk in primary care of colorectal cancer from new onset rectal bleeding: 10 year prospective study
}

\author{
Jennifer du Toit, William Hamilton, Kevin Barraclough
}

\begin{abstract}
Objective To measure the risk of colorectal cancer and adenoma with new onset rectal bleeding reported to primary care.

Design Cohort study.

Setting A rural general practice in the United Kingdom.

Participants Patients aged 45 or more with new onset rectal bleeding, irrespective of other symptoms.

Main outcome measures Percentage of participants in whom colorectal cancer or colonic adenoma was identified after investigation of the bowel.

Results During a 10 year period, 265 patients reported new rectal bleeding. Of these, 15 (5.7\%, 95\% confidence interval $3.2 \%$ to $9.2 \%$ ) had colorectal cancer, and $13(4.9 \%, 2.6 \%$ to $8.4 \%)$ had colonic adenoma. Only two of the patients with cancer had had diarrhoea.

Conclusions One in 10 patients aged 45 or more with new onset rectal bleeding had colonic neoplasia, so investigation of the bowel should be offered to all such patients, whether or not they have other symptoms.
\end{abstract}

\section{Introduction}

Rectal bleeding poses a problem for clinicians in primary care, because although it is common it can be a symptom of colorectal cancer. About $40 \%$ of patients with colorectal cancer have rectal bleeding, but the risk of colorectal cancer for a patient with rectal bleeding is thought to be relatively low. The main source of guidance for primary care in the United Kingdom, the NICE (National Institute for Health and Clinical Excellence) referral guidelines for suspected cancer, advise urgent referral of patients aged over 40 with six weeks of rectal bleeding accompanied by diarrhoea, and referral of patients aged 60 or more with rectal bleeding for six weeks without anal symptoms or diarrhoea. ${ }^{1}$

The risk of colorectal cancer in patients who present to primary care with rectal bleeding has rarely been studied. Most studies are retrospective: a Dutch study of 269 patients, nine of whom had cancer, found a positive predictive value of $3.3 \%$ (95\% confidence interval $1.2 \%$ to $5.4 \%$ ); a Belgian study of 386 patients, 27 of whom had cancer, found a value of $7.0 \%$ (4.6\% to 10.0\%); and a UK case control study of 2093 patients, 349 of whom had cancer, estimated a positive predictive value of $2.4 \%$ (1.9\% to $3.2 \%))^{2-4}$ A recent UK study investigated $219(69 \%)$ of 319 patients aged 34 or more whose main symptom was rectal bleeding. Eleven cancers were found, giving a positive predictive value of $3.4 \% .^{5}$ All patients with cancer in that study also had a change of bowel habit, mainly looser stools.

We report a prospective study of patients with new rectal bleeding carried out during more than a decade in a small but typical general practice.

\section{Methods}

In our rural practice (four doctors and one registrar and a mean list size of 4426 patients over the decade), we studied patients aged 45 or more who reported new rectal bleeding, irrespective of whether or not they also reported diarrhoea. A practice protocol established in 1993 required investigation of all patients aged 45 or more with a new episode of rectal bleeding, irrespective of duration or of possible anal causes. This policy was based on contemporary guidelines and was regularly reiterated. Investigation was by rigid sigmoidoscopy with barium enema (most patients), flexible sigmoidoscopy, or colonoscopy. JdT carried out all rigid sigmoidoscopies in the practice, usually within days of presentation, and the results were recorded at the same time. In the second half of the study period, direct access to flexible sigmoidoscopy became more available. Because of the convenience of the service provided and the small size of the practice, we believe that all patients aged 45 or more who presented with new rectal bleeding were investigated. To ensure that data collection was complete, each year we carried out a computer search for all patients who had had barium enemas, flexible sigmoidoscopies, or colonoscopies. This identified any patients who had been investigated but had not had a rigid sigmoidoscopy in the practice. We calculated the predicted number of colorectal cancers for the practice population by extrapolating the 2001 incidence figures for England and Wales. ${ }^{6}$

\section{Results}

During the 10 years and 3 months beginning January 1994, 38 colorectal cancers and 33 adenomas were diagnosed in practice patients, giving an overall annual incidence of cancer of 84/100 000 . The table shows results for rectal bleeding and the total number of colorectal cancers in patients from our practice. Rectal bleeding was accompanied by diarrhoea in two of the patients with cancer and four with adenoma.

\section{Discussion}

In primary care, a new episode of rectal bleeding in patients aged 45 or more had a positive predictive value for colorectal cancer of $5.7 \%$ (3.2\% to 9.2\%). This value was $4.9 \%(2.6 \%$ to $8.4 \%)$ for adenoma. Thus, one in 10 patients had colorectal neoplasia.

Our study is small and from a single practice, but it has the advantages that all patients with a new episode of rectal bleeding were investigated (as far as we can tell) and the data were collected prospectively. A small number of patients may not have entered our diagnostic protocol, despite frequent reminders to staff, so our

This article was posted on bmj.com on 21 June 2006: http://bmj.com/cgi/doi/10.1136/bmj.38846.684850.2F
Editorial by Weller

Hoyland House, Painswick,

Gloucestershire GL6 6RD

Jennifer du Toit general practitioner Kevin Barraclough general practitioner

Academic Unit of Primary Health

Care, Department of Community Based Medicine, University of Bristol, Bristol BS8 1AU

William Hamilton senior research fellow

Correspondence to:

$\mathrm{J}$ du Toit

kbarraclough@

ehotspot.co.uk

BMJ 2006;333:69-70 
Rectal bleeding and colorectal neoplasia in a prospective cohort of patients aged 45 or over in primary care

\begin{tabular}{|c|c|c|c|c|c|c|c|}
\hline \multirow[b]{2}{*}{ Age group } & \multirow{2}{*}{$\begin{array}{l}\text { Mean practice } \\
\text { population }\end{array}$} & \multirow{2}{*}{$\begin{array}{l}\text { No }(\%) \text { with new } \\
\text { rectal bleeding in } \\
10.25 \text { years }\end{array}$} & \multirow{2}{*}{$\begin{array}{l}\text { Incidence of rectal } \\
\text { bleeding/1000/year }\end{array}$} & \multicolumn{2}{|c|}{$\begin{array}{l}\text { No (\%) with colorectal neoplasia } \\
\text { and rectal bleeding }\end{array}$} & \multicolumn{2}{|c|}{$\begin{array}{l}\text { No with colorectal cancer (with or } \\
\text { without rectal bleeding) }\end{array}$} \\
\hline & & & & Cancer & Adenoma & Observed & Predicted $^{*}$ \\
\hline $45-54$ & 561 & 51 (9.1) & 8.9 & $2(3.9)$ & $0(0.0)$ & 3 & 2 \\
\hline $55-64$ & 762 & $75(9.8)$ & 9.6 & 1 (1.3) & $3(4.0)$ & 7 & 7 \\
\hline $65-74$ & 627 & $63(9.4)$ & 9.8 & $6(9.5)$ & $4(6.4)$ & 14 & 13 \\
\hline$\geq 75$ & 939 & $76(8.1)$ & 7.9 & $6(7.9)$ & $6(7.9)$ & 14 & 32 \\
\hline Total & 2889 & 265 (9.2) & 8.9 & $15(5.7) \dagger$ & 13 (4.9)‡ & 38 & 54 \\
\hline
\end{tabular}

${ }^{*}$ From incidence in England and Wales, $2001 .{ }^{6}$

t95\% confidence interval $3.2 \%$ to $9.2 \%$.

$\$ 95 \%$ confidence interval $2.6 \%$ to $8.4 \%$.

positive predictive values might be slightly overestimated. The observed total number of cancers in the practice was similar to the predicted number calculated from national incidence figures, apart from in the oldest age group, where fewer cancers occurred than were expected.

When positive predictive values from the three retrospective studies are recalculated for patients aged 50 or more, they are $11 \%(6.9 \%$ to $16.0 \%), 11 \%(5.1 \%$ to $21.0 \%)$, and $2.6 \%(2.2 \%$ to $3.0 \%))^{2-4}$ In the fourth study, $5.2 \%$ was quoted for patients aged 60 years or more. ${ }^{5}$ The lowest of these sets of values comes from a study that examined all rectal bleeding, whereas in the other three studies rectal bleeding was the main reason for consultation. The annual rate of new rectal bleeding in our population was $8.9 / 1000$, compared with 7-21/1000 in the Belgian and UK studies. ${ }^{3-5}$ The proportion of patients with colorectal cancer in our practice who presented with rectal bleeding was 39\%, which is similar to the proportion reported by the largest of the retrospective studies. ${ }^{4}$ These comparisons suggest that our figures are representative.

Only two of our patients with new rectal bleeding and cancer had accompanying diarrhoea. This contrasts with the findings of other studies, and it is important because rectal bleeding without diarrhoea does not qualify for an urgent referral under the current guidance, unless the patient is aged 60 or more, the bleeding persists for six weeks, and no anal symptoms exist. The risk of $5.7 \%$ for rectal bleeding on its own for colorectal cancer and $10.6 \%$ for all neoplasia suggests that new rectal bleeding in patients aged 45 or over should qualify for urgent referral, whether or not they have other symptoms.

The Department of Health's Referral Guidelines for Suspected Cancer published in July 2000 estimated the resources that would be needed to ensure that all patients with suspected cancer would be seen by a specialist within two weeks. ${ }^{7}$ This estimate was based on a population in which, for every case of colorectal cancer that was diagnosed, 15 other patients would be found to have benign disease. Thus, the population with suspected colorectal cancer referred using this guidance should have about a 7\% (1 in 16) absolute risk of having the condition. This implies that a symptom (or pattern of symptoms) with such a level of absolute risk warrants urgent referral. We believe that most patients would accept that the level of risk we found-even at our lower confidence interval of $3.2 \%$-is an adequate reason for investigation, and that current guidelines should be changed.

Thanks to the Bristol Bath Gloucestershire Primary Care Network.

\section{What is already known on this topic}

Estimated risks of cancer with rectal bleeding vary greatly (2.4-11\%), making it hard for doctors to advise patients on the need for investigation

\section{What this study adds}

In a prospective 10 year study in primary care, about one in 10 patients aged 45 or over with new onset rectal bleeding had colorectal neoplasia

All patients aged 45 or over with new onset rectal bleeding should be offered investigation of their bowel, whether or not they have other symptoms

Contributors: $\mathrm{KB}$ and JdT were involved in all aspects of the study. WH performed the analyses and wrote the initial draft $\mathrm{JdT}$ is guarantor.

Funding: WH is funded by his research practice (Barnfield Hill, Exeter) and an NHS researcher development award. Competing interests: None declared.

Ethical approval: Gloucester local research ethics committee.

1 National Institute for Health and Clinical Excellence. Referral guidelines for suspected cancer. London: NICE, 2005.

Fijten G, Starmans R, Muris J, Schouten H, Blijham G, Knottnerus JA Predictive value of signs and symptoms for colorectal cancer in patients Predictive value of signs and symptoms for colorectal cancer in pat
with rectal bleeding in general practice. Fam Pract 1995;12:279-86.

3 Wauters H, Van Casteren V, Buntinx F. Rectal bleeding and colorectal cancer in general practice: diagnostic study. BMJ 2000;321:998-9.

4 Hamilton W, Round A, Sharp D, Peters T. Clinical features of colorectal cancer before diagnosis: a population-based case-control study. $\mathrm{Br} \mathrm{J}$ Cancer 2005;93:399-405.

5 Ellis BG, Thompson M. Factors identifying higher risk rectal bleeding in general practice. BrJ Gen Pract 2005;55:949-55.

6 Update to Cancer Trends in England and Wales 1950-1999:National Statistics, 2003. www.statistics.gov.uk/downloads/theme health/CancerTrends 2003. www.statistics.gov.uk/down
Updates.pdf (accessed 9 May 2006).

7 Department of Health. Referral guidelines for suspected cancer. London: Department of Health. Referral guidelines for suspected cancer. London:
DoH, 2000. www.dh.govuk/PublicationsAndStatistics/Publications/ $\mathrm{DoH}, \quad 2000 . \quad$ www.dh.gov.uk/P
PublicationsPolicyAndGuidance/

PublicationsPolicyAndGuidanceArticle/fs/

en?CONTENT_ID = 4008746\&chk=jtsIsg (accessed 9 May 2006).

(Accepted 3 May 2006)

doi $10.1136 /$ bmj.38846.684850.2F

\section{Endpiece}

\section{Befriending illness}

If you can make a friend of your illness it will reward you a thousand-fold.

Native American saying.

Submitted by Geoffrey Marsh, retired general practitioner, Stockton on Tees 